\title{
Characterizing temporal genomic heterogeneity in pediatric high-grade gliomas
}

Ralph Salloum ${ }^{1 \dagger}$, Melissa K. McConechy ${ }^{2 \dagger}$, Leonie G. Mikael ${ }^{3 \dagger}$, Christine Fuller $^{1}$, Rachid Drissi ${ }^{1}$, Mariko DeWire ${ }^{1}$, Hamid Nikbakht ${ }^{2}$, Nicolas De Jay², Xiaodan Yang ${ }^{2}$, Daniel Boue ${ }^{4}$, Lionel M. L. Chow', Jonathan L. Finlay ${ }^{5}$, Tenzin Gayden², Jason Karamchandani ${ }^{6}$, Trent R. Hummel ${ }^{1}$, Randal Olshefski ${ }^{4}$, Diana S. Osorio ${ }^{4}$, Charles Stevenson ${ }^{1}$, Claudia L. Kleinman ${ }^{2,7}$, Jacek Majewski ${ }^{2}$, Maryam Fouladi ${ }^{1 *}$ and Nada Jabado ${ }^{2,3^{* \dagger}}$ (DD

\begin{abstract}
Pediatric high-grade gliomas (pHGGs) are aggressive neoplasms representing approximately 20\% of brain tumors in children. Current therapies offer limited disease control, and patients have a poor prognosis. Empiric use of targeted therapy, especially at progression, is increasingly practiced despite a paucity of data regarding temporal and therapydriven genomic evolution in pHGGs. To study the genetic landscape of pHGGs at recurrence, we performed whole exome and methylation analyses on matched primary and recurrent pHGGs from 16 patients. Tumor mutational profiles identified three distinct subgroups. Group $1(n=7)$ harbored known hotspot mutations in Histone $3(\mathrm{H} 3)$ (K27M or G34V) or IDH1 (H3/IDH1 mutants) and co-occurring TP53 or ACVR1 mutations in tumor pairs across the disease course. Group $2(n=7), \mathrm{H} 3 / \mathrm{IDH} 1$ wildtype tumor pairs, harbored novel mutations in chromatin modifiers (ZMYND11, EP300 $n=2)$, all associated with TP53 alterations, or had BRAF V600E mutations $(n=2)$ conserved across tumor pairs. Group 3 included 2 tumors with NF1 germline mutations. Pairs from primary and relapsed pHGG samples clustered within the same DNA methylation subgroup. ATRX mutations were clonal and retained in H3G34V and H3/ IDH1 wildtype tumors, while different genetic alterations in this gene were observed at diagnosis and recurrence in IDH1 mutant tumors. Mutations in putative drug targets (EGFR, ERBB2, PDGFRA, PI3K) were not always shared between primary and recurrence samples, indicating evolution during progression. Our findings indicate that specific key driver mutations in pHGGs are conserved at recurrence and are prime targets for therapeutic development and clinical trials (e.g. H3 post-translational modifications, IDH1, BRAF V600E). Other actionable mutations are acquired or lost, indicating that re-biopsy at recurrence will provide better guidance for effective targeted therapy of pHGGs.
\end{abstract}

Keywords: Pediatric high-grade gliomas, Recurrence, Genomics, Histone 3, ATRX, IDH1, NF1, Tumor evolution

\footnotetext{
* Correspondence: maryam.fouladi@cchmc.org; nada.jabado@mcgill.ca Ralph Salloum, Melissa K. McConechy and Leonie G. Mikael are co-first authors.

Maryam Fouladi and Nada Jabado are co-senior authors.

${ }^{\dagger}$ Equal contributors

'Brain Tumor Center, Cincinnati Children's Hospital Medical Center, 3333

Burnet Avenue, Cincinnati, $\mathrm{OH}$ 45229, USA

2Department of Human Genetics, McGill University, Montreal, QC H3A 1B1,

Canada

Full list of author information is available at the end of the article
} International License (http://creativecommons.org/licenses/by/4.0/), which permits unrestricted use, distribution, and reproduction in any medium, provided you give appropriate credit to the original author(s) and the source, provide a link to the Creative Commons license, and indicate if changes were made. The Creative Commons Public Domain Dedication waiver (http://creativecommons.org/publicdomain/zero/1.0/) applies to the data made available in this article, unless otherwise stated. 


\section{Introduction}

Genetic and epigenetic molecular profiling techniques have revolutionized our understanding of the etiology and biology of pediatric high-grade gliomas (pHGGs) (reviewed in [20]). Unfortunately, this has not yet led to an improvement in outcome for children with this disease [40] despite the use of agents that target pathways identified through these biological advances. Novel agents for the treatment of pHGGs are first tested in the relapse setting, and target genomic alterations typically present in therapy-naïve diagnostic tumor samples or models. However, there is limited data on the relevance of genomic aberrations at diagnosis on disease progression after multimodal therapy, making the effectiveness of this approach questionable. An improved understanding of temporal and therapy-driven evolution of recurrent pHGGs is therefore needed, especially in the context of hemispheric HGGs that show increased genetic heterogeneity $[5,12,13,19,37,50,51]$.

Clonal evolution is a dynamic process that has been reported in many cancer types [3, 28, 39, 48], even without exposure to therapy [11]. Morrissy et al., have recently demonstrated poor overlap in genetic events between primary and post-treatment medulloblastoma both in murine models and human samples [28]. This included a marked divergence in actionable genes between diagnosis and recurrence, despite conservation of molecular subgroup affiliation [28, 36, 47]. Whole exome sequencing (WES) of 23 initial and recurrent gliomas in adults by Johnson et al., revealed variable genetic relatedness across pairs; in 10 cases, most mutations from diagnosis were not conserved in the recurrent sample, including the $B R A F$ V600E hotspot mutation [19]. In adult glioblastoma multiforme (GBM), a longitudinal study of the genetic landscape of 114 untreated and recurrent paired tumors revealed a switch in expression-based subtypes in $63 \%$ of cases. Enrichment of a hypermutated phenotype in recurrent disease exposed to temozolomide (TMZ) was also identified, suggesting the occurrence of therapy-induced mutagenesis [45]. Moreover, an analysis of tumor phylogeny revealed that dominant clones at recurrence were infrequently direct descendants of dominant clones from diagnosis [45]. We have previously shown that disease-defining somatic mutations in oncohistones [K27M in Histone $3(\mathrm{H} 3)$ variants $(H 3 F 3 A, H I S T 1 H 3 B)]$ are spatially stable in diffuse intrinsic pontine glioma (DIPG), and co-occur with highly conserved partners throughout geographically distinct tumor sites [18, 30]. However, limited data on disease recurrence are available for supratentorial pHGGs. This is of major therapeutic interest as hemispheric pHGGs show more genetic variability at diagnosis than midline tumors, the vast majority of which are defined by H3K27M mutations (>90\%) $[14,51]$. In the current study, we characterize the temporal genomic heterogeneity in pHGGs by assessing the mutational profile and methylome of paired primary and recurrent tumors with emphasis on supratentorial pHGGs.

\section{Materials and methods Clinical cohort}

Institutional review board approval was obtained to perform this retrospective study at Cincinnati Children's Hospital Medical Center (CCHMC, Study ID: 20146849) and Nationwide Children's Hospital (NCH: IRB1500143). The patient cohort was chosen based on the availability of material from both the primary and recurrent tumor for each case with a confirmed HGG diagnosis Two neuropathologists (CF and JK) independently reviewed tumor samples. Patient tumor samples were acquired from diagnosis as well as recurrence or autopsy and preserved either as fresh-frozen or formalin fixed paraffin embedded (FFPE) tissue. Blood or other matched normal tissue was obtained when available for germline analysis. To ensure adequate tumor content, hematoxylin and eosin $(\mathrm{H} \& \mathrm{E})$ slides were reviewed from each frozen specimen, the initial cut of each FFPE block, and an additional cut of FFPE block after scrolls were obtained for DNA extraction. All patient tumor and matched blood samples were collected after informed consent was provided by patients or legal guardians through institutional review board approved protocols at the respective institutions.

\section{DNA extraction}

DNA extraction was carried out from frozen tissue using the Qiagen AllPrep DNA/RNA/miRNA Universal Kit following the manufacturer's instructions. DNA from FFPE scrolls or core punches were isolated by suspending the paraffin scrolls in deparaffinization solution (Qiagen) followed by DNA extraction using the QIAamp DNA FFPE Tissue Kit. DNA quantification was conducted using the Quant-iT Picogreen dsDNA assay kit (Thermo Fisher Scientific). Droplet digital PCR (ddPCR) assays for H3K27M mutations were performed as previously described [30].

\section{Whole Exome Sequencing (WES) analysis}

The Nextera Rapid Capture Exome kit (Illumina) was used to prepare 36 libraries, and the Agilent SureSelect Reagent Exome kit (Agilent) was used to prepare 6 libraries according to the manufacturer's instructions. Genomic DNA was extracted from frozen tissue and FFPE blocks representing tumor or normal tissue and from monocytes. Sequencing was performed on the Illumina HiSeq 2000 using rapid-run mode with $100 \mathrm{bp}$ paired-end reads. Adaptor sequences were removed, and reads trimmed for quality using the FASTX-Toolkit (http://hannonlab.cshl.edu/fastx_toolkit/). An in-house 
program was used to ensure the presence of exclusively paired-reads. We next aligned the reads using BurrowsWheeler Aligner (BWA) 0.7.7 to GRC37/hg19 as a reference genome. Indel realignment was performed using the Genome Analysis Toolkit (GATK) 29 (https://software.broadinstitute.org/gatk/). Duplicate reads were marked using Picard (http://broadinstitute.github.io/picard/), and excluded from further analyses. The average coverage for all the samples was 69X. Single Nucleotide Variants (SNVs) and short indels were called using our in-house pipeline that exploits three different variant callers: FreeBayes 1.1.0 (https://arxiv.org/abs/1207.3907), SAMtools 1.3.1 (http://samtools.sourceforge.net/) and GATK HaplotypeCaller 3.7 [43]. Thresholds were set for calling a true variant to two out of three variant callers. Next, variants were filtered for quality so at least $10 \%$ of reads supported each variant call. ANNOVAR [46] and in-house programs were used to annotate variants that affect protein-coding sequence. Variants were screened to assess whether they had previously been observed in public datasets including the 1000 Genomes Project data set (November 2011), the National Heart, Lung and Blood Institute (NHLBI) Grand Opportunity (GO) exomes as well as in over 3000 exomes previously sequenced at our center (including cancer and non-cancer samples).

\section{Somatic and putative somatic mutation identification from Whole Exome Sequencing}

Protein coding variants were identified as nonsynonymous missense, frameshifts, stopgains, indels, splice variants, and present with a minimum of $5 \%$ mutant allele frequency (MAF) or greater. A MAF of $>5 \%$ was used in order to reduce removal of low frequency mutations in genes of interest (H3F3A, TP53, ATRX, ZMYND11, LZTR1). All other reported mutations have a MAF > 15\%. To be considered somatic, zero mutant variant reads were present in the normal sample. In some cases where $<2$ variant reads were present in the normal, the sequencing alignments were manually checked to verify as sequencing artifacts. Additionally, all somatic and putative somatic variants were manually checked for alignment and sequencing artifacts. To further remove germline SNPs where normal was not available, all samples were filtered based on a MAF (mutant allele frequency) < 0.0005 in the 1000 Genomes Project (November 2011), EVS, and $<0.00005$ from ExAC databases. Finally, in cases with no associated normal, variants were only reported when present in COSMIC or high/medium functional impact as assessed using MutationAssessor (http://mutationassessor.org/r3/). Targeted validations on selected variants were performed using Sanger sequencing or high-depth sequencing on an Illumina Miseq as previously described [30].

\section{Allelic Imbalance and CNV analysis}

Allelic Imbalance was assessed in whole exome sequencing data using the ExomeAI program [29] for 15 pHGG tumor pairs using the default parameters of the program. HGG1 was not analyzed due to low WES coverage of the recurrence tumor. Copy number variations (CNVs) were analyzed in 8 pHGG tumor-normal pairs using an in-house program (CNAXX; unpublished) we developed that takes both coverage (normalized average) and the deviation of B allele frequency from $50 \%$ into account (adapted from methods used in FishingCNV [38] and ExomeAI [29]). Different CNV events (amplification, deletion and copy neutral $\mathrm{LOH}$ ) were called based upon the status of the normalized coverage and the $\mathrm{B}$ allelic imbalance as we described previously in [30]. We assessed the CNV events at both the chromosomal arm level and the union of the segments called by each of the two features used by the program (i.e. B allele frequency and normalized average coverage). Paired t-tests were performed using the statistical program $\mathrm{R}$ version 3.1.1.

\section{DNA methylation analysis}

Illumina $450 \mathrm{~K}$ methylation chips were used on 28 samples, and profiling data was analyzed as previously described $[14,32]$. In some cases, only the tumor from the primary (HGG1, HGG3, HGG11) or recurrence (HGG12) were included in the final analysis due to insufficient or poor quality FFPE DNA from the respective tumor pair. The raw data were subject to quality control and preprocessing utilizing the $\mathrm{R}$ package minfi, and normalized for technical variation between the Infinium I and II probes using the SWAN method. We removed probes on sex chromosomes (chr X, Y), those containing SNPs (dbSNP: https://www.ncbi.nlm.nih.gov/projects/SNP/) as well as non-specific probes that bind to multiple genomic locations. Unsupervised hierarchical clustering was performed using average linkage, and Pearson rank correlation distance on the top 3000 most variable probes selected based on standard deviation of beta values $(\beta$ values).

\section{Immunohistochemistry for MMR proteins}

Immunohistochemistry (IHC) staining for expression of MMR proteins (MLH1, MSH2, MSH6, PMS2) was performed on slides cut from FFPE blocks of pHGG samples using conventional methods [53].

\section{Results}

\section{Patient characteristics}

Paired primary and recurrent pHGG tumor samples were available from 16 patients. Median age at diagnosis was 15 years (range: 4-29 years). Among the 16 cases, one patient with a germline NF1 mutation was initially diagnosed with a WHO grade II glioma with pilocytic 
features (HGG16) before recurring as a HGG. Median time to progression was 13 months (range: 4-45 months). Fourteen patients received radiation therapy at diagnosis, and 10 patients $(63 \%)$ had received temozolomide (TMZ) prior to last progression. Five tumors (31\%) were midline [2 pontine tumors (HGG2, HGG4), 1 thalamic tumor (HGG8), 2 spinal tumors (HGG1, HGG3)], and 11 tumors (69\%) were hemispheric. Two patients (HGG15 and HGG16) were previously known to have germline neurofibromatosis type 1 (NF1). All clinicopathological features are shown in Table 1.

\section{Core oncogenic mutations are shared in primary and recurrent pHGGs}

To determine the temporal stability of the mutational landscape of pHGGs, WES was performed on all 16 tumor pairs. Matched germline DNA was available for 9 patients. Samples were sequenced with an average coverage of 69X (range 3.5-200.4X) (Additional file 1: Table S1). Tumor pairs were stratified into three distinct molecular groups based on the identified mutation patterns (Fig. 1a, Additional file 2: Table S2). The first group was comprised of H3/IDH1 mutant tumors (7/16 pairs, 44\%), where both the primary and recurrence harbored shared epigenetic driver mutations in H3F3A K27M ( $n=3$, HGG1, HGG2, HGG3), HIST1H3B K27M ( $n=1$, HGG4), H3F3A G34V ( $n=1$, HGG5), or IDH1 R132H/S ( $n=2$, HGG6, HGG7). Consistent with previous studies, all H3K27M mutant tumors were in the brain midline (spine and pons), while H3F3A G34 V and IDH1 mutant tumors were hemispheric $[37,41]$. In addition, all six $H 3 F 3 A$ and $I D H 1 \mathrm{mu}-$ tant tumors had co-occurring TP53 mutations while the HIST1H3B (Histone 3.1) K27M mutant DIPG had a known associated ACVR1 R258G mutation (HGG4) [14, 42, 51]. H3K27M/G34V and IDH1 mutations were as expected clonal and conserved across primary and recurrent tumor pairs. Similarly, ACVR1 and TP53 mutations, which have been shown to be obligate partners in $\mathrm{H} 3$ and IDH mutagenesis, were also conserved throughout disease progression.

The second group had no identifiable $\mathrm{H} 3$ or IDH1 mutations, termed H3/IDH1 wildtype (7/16 pairs, 44\%). This is a heterogeneous group of supratentorial, mostly hemispheric, tumors $(6 / 7,86 \%)$ with clonal mutations identified in TP53 (4/7, 57\%), ATRX (3/7, 43\%), BRAF V600E (2/7, 29\%), BCOR (1/7, 14\%), CDKN2A (1/7, $14 \%), Z M Y N D 11(1 / 7,14 \%)$, and EP300 (1/7, 14\%) in tumor pairs (Fig. 1a). HGG8, the only H3/IDH1 thalamic wildtype tumor, had TP53 missense, ATRX missense, CDKN2A nonsense, and interestingly, EP300 missense mutations that were retained at recurrence. One sample (HGG11) had a hypermutated phenotype with 151 protein coding somatic mutations at diagnosis and 670 at relapse. The H3/IDH1 wildtype group was enriched for multiple mutations in receptor tyrosine kinase (RTK), phosphoinositide 3-kinase (PI3K) pathway, histone modifiers, integrin, and cadherin genes (Fig. 1a, b). Except for rare mutations, most of these genetic alterations were not present across tumor pairs, and seemed to occur more frequently at disease recurrence (Fig. 1a, b).

Lastly, the third molecular group was composed of patients with NF1 germline truncating mutations $(2 / 16$, 12\%) with associated HGG (HGG15, HGG16). There were few SNVs identified in the primary tumors from these 2 cases. The primary tumor of HGG15 had no detectable driver SNVs, however, it acquired an ATRX frameshift mutation as well as a RAD50 missense mutation at recurrence (Fig. 1b). In HGG16, both primary and recurrent samples harbored PPM1D nonsense (L513X) and ATRX missense mutations. Previous studies have shown that PPM1D mutations affect p53 function and are mutually exclusive of TP53 mutations [55]. This mutation pattern in the NF1 mutant group is reminiscent of G34R/V-H3F3A mutated HGGs which co-occur with $A T R X$ and TP53 alterations.

\section{Other mutational patterns at diagnosis and recurrence}

In all groups of pHGG tumors, core oncogenic driver mutations in $H 3$ variants, IDH1, TP53, ACVR1, BRAF $V 600 E$, and $P P M 1 D$ were conserved at recurrence. As expected, the TP53 pathway (TP53 or PPM1D) was the most frequently altered $(11 / 16,69 \%)$ across all tumor subgroups (Fig. 1a). ATRX mutations were also frequent $(8 / 16,50 \%)$ and, as previously described, were enriched in supratentorial samples $(7 / 10,70 \%)[1,9,14,26]$. In the H3/IDH1 mutant group, ATRX mutations were observed in 3 hemispheric tumor pairs: one pair with H3G34V (HGG5), and two with IDH1 R132H/S (HGG6, HGG7) mutations. Additionally, ATRX mutations were identified in three H3/IDH1 wildtype tumor pairs, two of which co-occurred with alterations in chromatin modifiers (HGG8, HGG9), and in both samples from patients with germline NF1. We observed the same ATRX mutation at recurrence in all tumor pairs of the $\mathrm{H} 3$ / IDH1 wildtype subgroup. In IDH1 mutant tumors, HGG7 harbored two ATRX alterations including a frameshift mutation exclusive to the primary tumor, while HGG6 acquired an additional ATRX missense mutation in the recurrence, in keeping with previous findings in IDH1 mutagenesis [19]. While taking into consideration the limitations imposed by our relatively small cohort, our findings indicate that in H3/IDH1 wildtype pHGGs, the same ATRX mutation, when present at diagnosis, is retained at disease recurrence despite gross total resection of the primary tumor in most cases. Interestingly, while loss of $B R A F \mathrm{~V} 600 \mathrm{E}$ at recurrence has been reported in adult gliomas [19], the two cases in our study (HGG12, HGG13) retained this mutation at recurrence. 


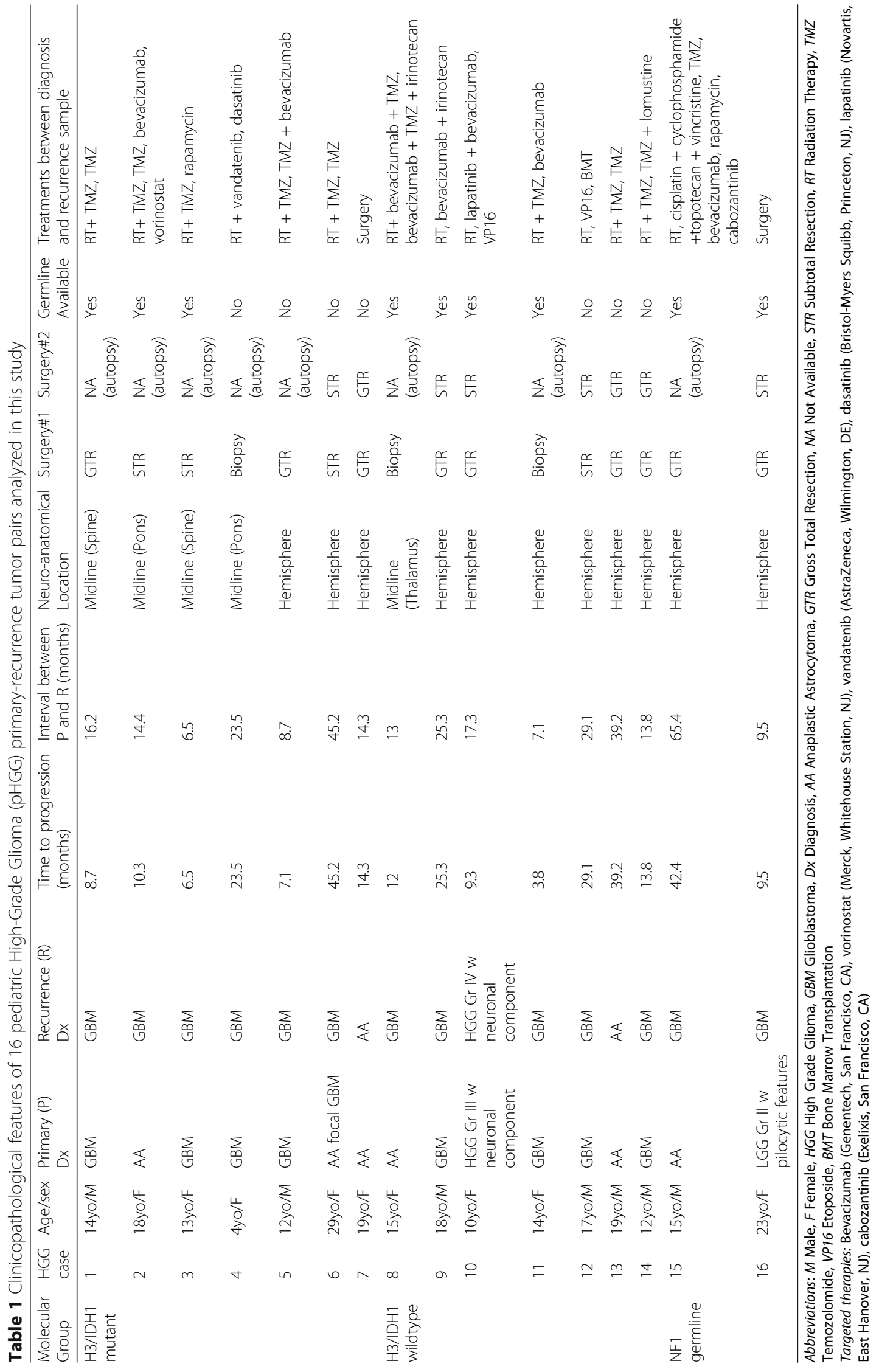




\begin{tabular}{|c|c|c|c|c|}
\hline $\mathbf{a}_{\text {Group/Case ID }}$ & 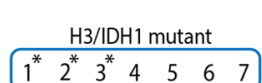 & 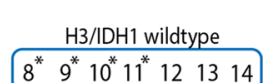 & $\begin{array}{l}\text { NAlpline } \\
\text { germing } \\
115^{*} 16^{*}\end{array}$ & 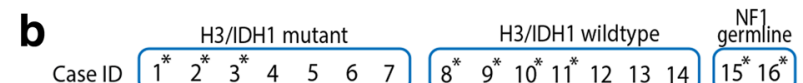 \\
\hline Age/Sex & $14 \mathrm{M} 18 \mathrm{FF} 13 \mathrm{~F} \quad 4 \mathrm{~F} 12 \mathrm{M} 29 \mathrm{~F} 19 \mathrm{M}$ & $15 F 18 M$ IOF $14 F 17 \mathrm{M} 19 \mathrm{M} 12 \mathrm{M}$ & $15 \mathrm{M} 23 \mathrm{~F}$ & Histone 1 \\
\hline & & & & 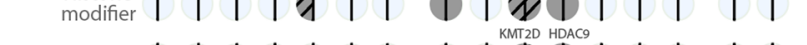 \\
\hline $\mathrm{H} 3 \mathrm{~F} 3 \mathrm{~A}$ & 19 & & & 111111111100111111 \\
\hline $\begin{array}{r}\text { HIST1H3B } \\
\text { IDH1 }\end{array}$ & 1010 & & & 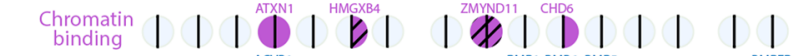 \\
\hline & i i i i i i & (1) & 0 & ACVR1/BMP $|+|$ \\
\hline & & & & 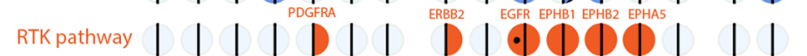 \\
\hline PPM1D & & & 10 & 111111111110 \\
\hline $\begin{array}{r}\text { CDKN2A } \\
\text { ATRX }\end{array}$ & & 0 & 1 & $1111111111 \geqslant 10111$ \\
\hline ZMYND11 & & 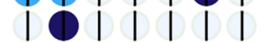 & & 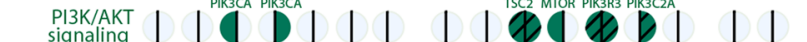 \\
\hline $\begin{array}{r}\text { EP300 } \\
\text { Histone modifier }\end{array}$ & & (1) & & 1111111111011111 \\
\hline Chromatin binding & 110 & 11 & 11 & $\underset{\text { Depair }}{\text { DNA break }}||||||||||||||$ \\
\hline $\begin{array}{l}\text { BCOR } \\
\text { ACYR1 }\end{array}$ & & & & 11111111111101110 \\
\hline & $\begin{array}{llll}1 & 1 & 1 & 1\end{array}$ & 110011 & 10 & 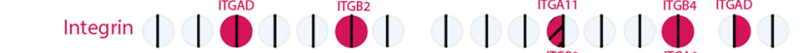 \\
\hline $\begin{aligned} \text { RTK genes } \\
\end{aligned}$ & 111101 & 101 & & 11111111111011011 \\
\hline $\begin{array}{l}\text { PISK/AK signaling } \\
\text { DNA break repair }\end{array}$ & $\begin{array}{lllll}1 & 1 & 1 & 1 & 0 \\
1 & 1 & 1 & 1\end{array}$ & 111011 & DI & 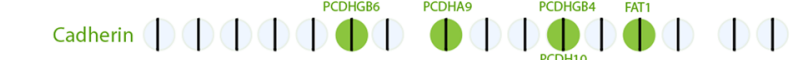 \\
\hline $\begin{array}{l}\text { Integrin } \\
\text { Cadherin }\end{array}$ & 110110 & & i & 11111111110111 \\
\hline SLITRK genes & & 1110 & & 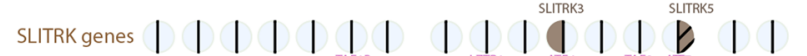 \\
\hline Transcription & 11 & Di & 11 & Transcription $111|1|$ \\
\hline & Spine Thalamus & Missense mutation & & \\
\hline & Pons Hemisphere & Truncating mutation & & rimary tumor \\
\hline & Primary tumor & $\begin{array}{c}\text { Additional or different su } \\
\text { missense mutation prese }\end{array}$ & & Decurrent tumor \\
\hline & Recurrent tumor & $\begin{array}{l}\text { Additional or different tsu } \\
\text { truncating mutatition pres }\end{array}$ & & \\
\hline & * Normal available & & & \\
\hline $\begin{array}{l}\text { Fig. } 1 \text { Mutationa } \\
\text { study. Vertical co } \\
\text { right half represe } \\
\text { half-colored circle } \\
\text { mutated. Mutatio } \\
\text { age, sex and tun } \\
\text { (frameshift or no } \\
\text { showing individu }\end{array}$ & $\begin{array}{l}\text { al profile of primary a } \\
\text { olumns of circles repr } \\
\text { ents the recurrence. } \\
\text { e indicates a mutation } \\
\text { ons shown are known } \\
\text { mor location are indi } \\
\text { onsense), orange = a } \\
\text { lual genes mutated i }\end{array}$ & $\begin{array}{l}\text { ecific to } \\
\text { be path } \\
\text { ed for }\end{array}$ & & $\begin{array}{l}\text { alyzed in this } \\
\text { tumor and the } \\
\text { ttation, and a } \\
\text { athways that are } \\
\text { lecular group, } \\
\text { ue = truncating } \\
\text { ach gene group } \\
\text { ations are shown }\end{array}$ \\
\hline
\end{tabular}

\section{Novel epigenetic alterations in H3/IDH1 wildtype pHGGs}

Two tumor pairs in the H3/IDH1 wildtype group showed potentially novel epigenetic drivers that converge to affect the same histone mark directly affected in pHGGs carrying H3.3 G34R/V or H3K27M mutations. Both primary and recurrent samples from HGG9, an adolescent patient with a parietal brain tumor, shared a ZMYND11 frameshift mutation that possibly abrogates expression of this protein, and they also harbored concurrent TP53 and ATRX missense mutations. These characteristics are typical of pHGGs that harbor H3.3G34R/V mutations. ZMYND11/BS69 has been shown to specifically recognize $\mathrm{H} 3 \mathrm{~K} 36 \mathrm{me} 3$ to regulate and repress transcription [17, 49]. Conversely, H3.3G34R/V mutant nucleosomes affect the trimethylation of $\mathrm{K} 36$ on the H3.3 mutant nucleosomes [24], impairing the recognition of H3K36me3 ZMYND11/BS69 and its action on modulating translation [49]. In HGG8, both primary and recurrent thalamic tumors shared a mutation in EP300 (D1339N), concurrent with TP53 and ATRX missense mutations and a CDKN2A nonsense mutation. The EP300 gene encodes a histone acetyltransferase (HAT), with acetylation activity at H3K27, and has been shown to regulate transcription through multiple mechanisms of chromatin remodeling [33]. The D1339N mutation has been identified in multiple tumor types, but not in HGG, and is presumed to affect the enzymatic activity of the protein $[8,25]$. It is worth noting that HGG8 harboring mutant EP300 was 
a midline tumor, a location where H3K27M mutations known to affect H3K27 acetylation usually account for the vast majority of driver mutations. Taken together, these data suggest that both these novel epigenetic mutations are relevant in the setting of pHGGs, and may possibly mimic the effect of genetic drivers in $\mathrm{H} 3$ mutant tumors.

\section{Activating mutations of the RTK-PI3K pathway are not always conserved}

Mutations in the RTK genes involving EGFR, ERBB2, $E R B B 4, F L T 1$ and $E P H A / B$, were identified in $5 / 7$ (71\%) of the H3/IDH1 wildtype tumor pairs. Many of these were exclusive to the primary or recurrent sample (Fig. 1b). In the H3/IDH1 mutant tumor HGG5, a PDGFRA Y288C missense mutation was acquired at recurrence. Similar to previous reports, PI3K mutations were temporally heterogeneous [30], where $1 / 5$ tumor (20\%) exhibited a shared mutation in a PI3K regulatory subunit PIK3R3 (HGG12). Additionally, subclonal mutations in the PI3K catalytic subunit were private to the primary tumor only in one case (HGG3) and were acquired at recurrence in two cases (HGG4, HGG13). Prior to our study, testing of a different tumor sampling from HGG3 was performed using a clinical genomic panel. This analysis identified a low frequency PIK3CA H1047R hotspot mutation that was not found in either the WES analysis of a different primary tumor tissue block or targeted high-depth sequencing of multiple samplings of the recurrent tumor (Additional file 3: Figure S1). In the hypermutated HGG11 tumor pair, the primary tumor harbored a missense $M T O R$ mutation, while at recurrence the tumor acquired a PI3K catalytic subunit PIK3CD passenger mutation (Fig. 1b).

\section{Mutational burden, allelic imbalance and copy number variations}

Analysis of the mutational burden showed no statistically significant difference in the number of mutations between primary and recurrent tumors across all groups (paired t-test, $p=0.24$ ) (Fig. 2a, Additional file 4: Table S3, Additional file 5: Figure S2). It is worth noting that within the limitations of sample size, we observed a trend towards an increase in the mutational burden at recurrence that did not reach statistical significance despite the use of TMZ as adjuvant therapy in 10/16 (63\%) pHGGs. In HGG11, we observed a marked increase in the number of somatic mutations in the primary $(n=151)$ and at recurrence $(n=670)$ compared to all other tumor samples, indicating a hypermutated phenotype. We identified and validated a germline $M L H 1$ splice missense mutation, and also performed immunohistochemistry on MMR proteins (MLH1, MSH6, MSH2, PMS2) on the primary HGG11 tumor (Additional file 6: Figure S3). Although IHC results did not show loss of any MMR proteins, we hypothesize that the splice mutation that translated extra inframe amino acids (data not shown), resulted in a dysfunctional yet nuclear-localized MLH1 protein. This may explain MMR IHC nuclear positivity in the setting of mismatch repair deficiency resulting in hypermutation. Interestingly, the mutation burden in that case dramatically increased at recurrence, which may be attributable to the combined effects of radiation and TMZ treatment [45].

To further assess chromosomal alterations in all of the primary-recurrent tumor pairs, we used WES data to analyze the state of allelic imbalance using ExomeAI [29]. Copy Number Variations (CNVs) were analyzed in eight tumor pairs with available matched normal. We calculated the numbers of allelic imbalance regions as
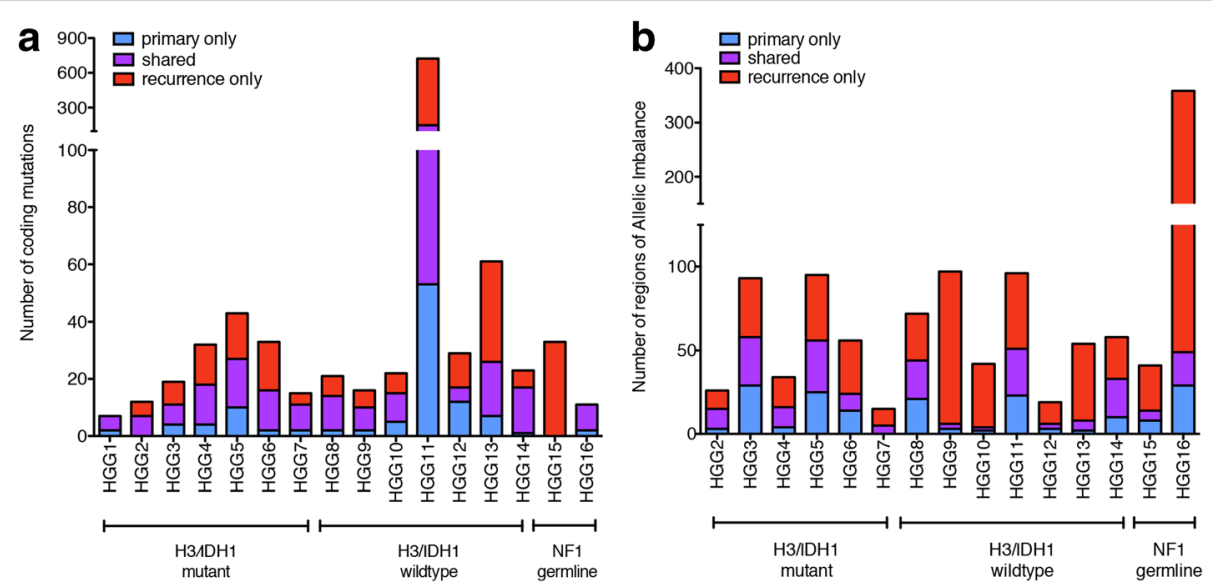

Fig. 2 Number of mutations (a) or regions of allelic imbalance (b) calculated by ExomeAl [29] specific to the primary tumor (blue), recurrence $($ red), or shared (purple) in the pHGG tumor pairs analyzed in this study. See also Additional files 2 and 8: Tables S2 and S4 
shared or specific to the primary or recurrent tumor (Fig. 2b, Additional file 4: Table S3), regardless of the size of each region. Similar to mutation counts, there was no significant difference in the number of regions of allelic imbalance between the primary and recurrent tumors across all subgroups (paired t-test, $p=0.071$ ). One tumor pair, HGG9, was particularly remarkable as there was an increased number of allelic imbalance regions in the recurrent tumor compared to the primary. Assessment of copy number variations confirmed genomewide loss of heterozygosity events at recurrence resulting in a copy number neutral genome (Additional file 7: Figure S4), compatible with radiotherapy-induced chromosomal alterations $[22,54]$. Both $N F 1$ germline cases also showed an increase in the number of regions of allelic imbalance. In both $N F 1$ germline recurrent tumors, CNV analysis showed loss of heterozygosity in the whole $\mathrm{q}$ arm of chromosome 17, containing the NF1 gene locus (Additional files 8 and 9: Tables S4 and S5). Our limited $\mathrm{CNV}$ analysis did not show any focal deletions or amplifications in genes of interest (PDGFRA, MYC, CDKN2A/B, $C D K 6)$ that have been previously implicated in pHGG [37]. In 5 of 6 tumors with TP53 mutations, large copy number alterations in the $\mathrm{p}$ arm of chromosome 7 were present that included the TP53 gene loci (Additional file 8: Table S4).

\section{DNA methylation subgroup is maintained at recurrence in PHGG}

We performed unsupervised clustering of DNA methylation in tumor pairs integrated into a larger pHGG inhouse data set (Fig. 3). As expected, tumors in the H3/ IDH1 mutant group clustered within their respective methylation cluster, while H3/IDH1 wildtype and NF1 germline groups clustered with H3/IDH1 wildtype HGGs. In all cases, the recurrent tumors clustered within the same methylation subgroup, similar to findings in other brain tumors including DIPG and medulloblastoma [30, 31].

\section{Discussion}

In this work, we performed whole exome sequencing on 16 primary and recurrent pHGG pairs including two pHGGs from patients with germline NF1 mutations, and provide insight into the temporal genomic evolution of these tumors. A direct comparison of the mutational landscape of paired samples reveals that oncogenic

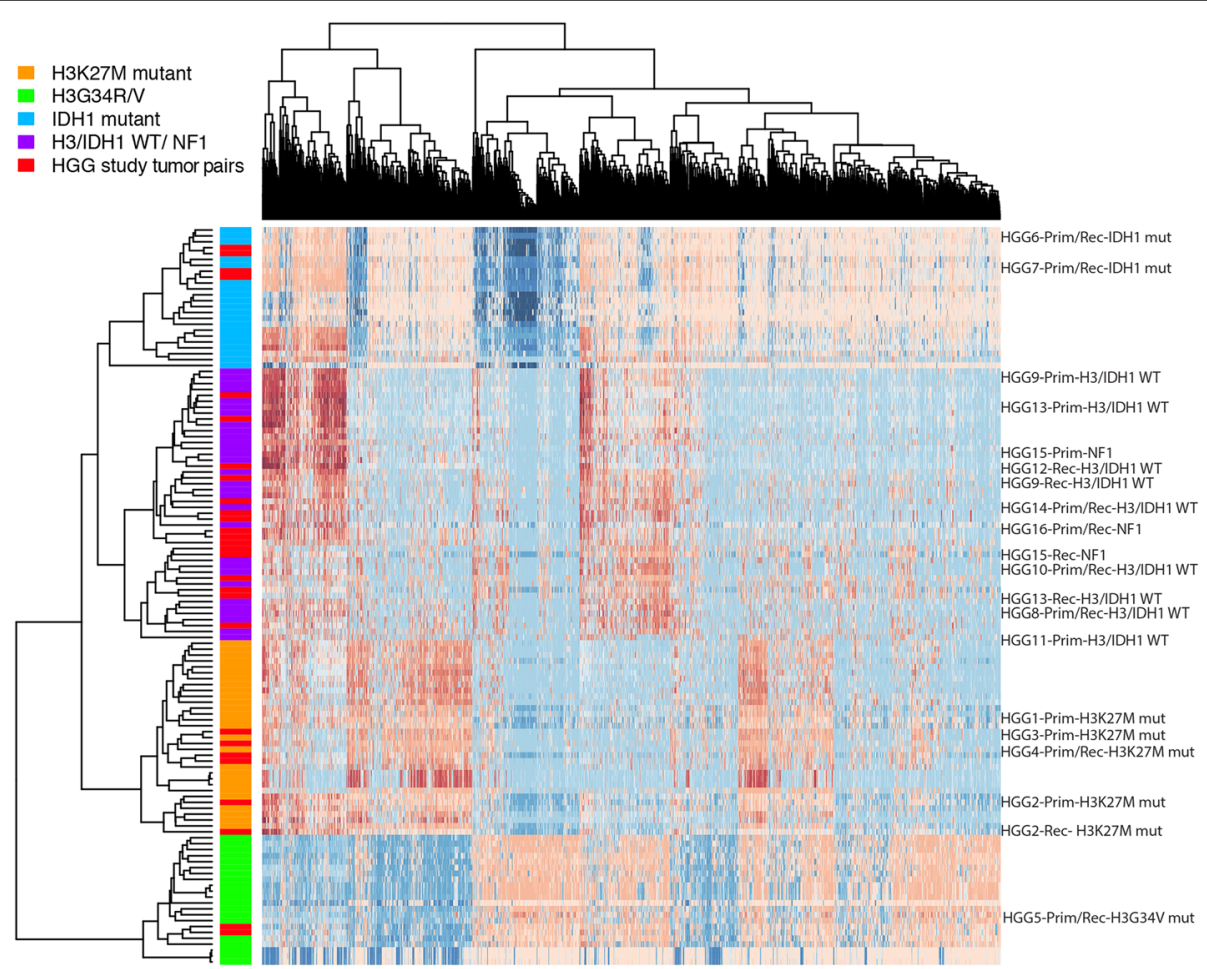

Fig. 3 Methylation heatmap of pHGG tumors analyzed in this study. Primary and/or recurrent tumors from 16 pHGG samples from this study were analyzed with a large in-house dataset using $450 \mathrm{~K}$ methylation probes for clustering. In specific cases, tumor DNA from the primary only (HGG1, HGG3, HGG11) and recurrence only (HGG12) were available. Red bars within the colored group on the left represent the clustering of pHGG tumor samples within the known pHGG molecular group. Methylation groups are represented by colors: orange $=\mathrm{H} 3 \mathrm{~K} 27 \mathrm{M}$, green $=\mathrm{H3} 334 \mathrm{RN}$, blue $=\mathrm{IDH} 1$ mutant, purple $=$ histone wildtype (WT). HGG case IDs for each tumor are indicated on the right. The label Prim/Rec indicates the clustering of both the primary and recurrence together. When the clustering location is different for the primary and recurrence tumor, the label is indicated as Prim (Primary) or Rec (Recurrence) 
driver mutations are typically conserved. The identification of these mutations in both the primary and recurrent tumors suggests that these mutations are early initiating events in tumorigenesis, are stable, and unaffected by treatment. This is in contrast to adult GBM where cancer driver mutations can be subclonal in the primary and recurrent tumors [19].

In our dataset, 10 of 16 patients were treated with TMZ, and despite a trend towards an increase in the number of mutations at recurrence, there was no statistically significant increase in mutational burden. This is in contrast to adult GBM, where an increase in mutational burden is observed with TMZ treatment [19]. Although our sample size is small, our findings may reflect a different biological process in response to TMZ in children compared to adults and warrants further evaluation. We observed one H3/IDH1 wildtype primary tumor (HGG11) with an increased number of somatic mutations compared to other primary tumors, and was identified to harbor a germline $M L H 1$ splice missense mutation. Immunohistochemical analysis did not show loss of the MMR proteins, however, we hypothesize that the missense splice mutation likely resulted in the translation of a dysfunctional MLH1 protein product to cause mismatch repair deficiency (MMRD) and hypermutation. After treatment with radiation and TMZ, this tumor acquired an increased number of somatic mutations compared to the primary tumor, suggesting that treatment further exacerbated the hypermutated phenotype. Several controversial and contradictory studies have variably reported the presence of microsatellite instability which results in mismatch repair deficiency in pediatric HGG and adults [10,44], highlighting the need for further studies. Future genetic testing for MMRD in pediatric HGG patients could steer treatment towards immunotherapy, as immune checkpoint blockade has shown clinical benefits in MMRD colorectal cancers as well as children with high-grade glioma $[4,23]$.

Similar to findings in adult IDH1-mutant gliomas [19], we identify heterogeneous ATRX alterations among IDH1 mutant pHGG tumor pairs. While IDH1 mutant tumors are more common in adult GBM and occur in up to $98 \%$ of secondary GBMs, they make up less than $10 \%$ of all pediatric HGGs $[2,52]$. In contrast to IDH1mutant gliomas, ATRX mutations associated with H3G34V, ZMYND11, EP300, or BRAF V600E were stable across the disease course in our study. Additionally, the BRAF V600E mutation was present in both primary and relapse samples in two children in our study which is in contrast to adult studies where it was identified either at diagnosis or at recurrence [19].

H3/IDH1 wildtype pHGGs have previously been shown to be a diverse group of tumors with mutations in many cancer pathways $[35,37,51]$, but have not been directly linked to any particular epigenetic driver as is the case with $\mathrm{H} 3$ and IDH1 mutant tumors. Our data reflect the heterogeneity of tumors in the H3/IDH1 wildtype group while also identifying two novel pHGG epigenetic cancer drivers (ZMYND11 and EP300) in this group. ZMYND11 has recently been described as an epigenetic regulator that specifically interacts with H3K36me3 to regulate transcription. Wen et al. have reported that $\mathrm{H} 3 \mathrm{G} 34 \mathrm{R} / \mathrm{V}$ mutations impair binding of ZMYND11 to an H3.3K36me3 peptide, suggesting that H3.3 G34R/V and ZMYND11 mutations alter H3K36me3 levels in similar fashions [49]. To the best of our knowledge, ZMYND11 mutations have not been previously described in pHGGs. The tumor harboring this mutation (HGG9) was located in the right parietal lobe and carried partner mutations in ATRX and TP53, further supporting its similarity to hemispheric H3.3 G34R/V mutated tumors. In addition, inactivating mutations identified in the HAT gene EP300 have been implicated in a wide array of cancer types including diffuse large B cell lymphoma [34], head and neck, esophageal, colorectal, medulloblastoma and non-small cell lung carcinoma [7, 15]. We also report a specific EP300 hotspot D1399N mutation (HGG8) which has not been previously identified in HGGs. Structural analysis of EP300 has shown that the D1399 residue has effects on the conformation of the HAT domain, specifically the L1 loop [25]. This is also an inactivating mutation which abolishes autoacetylation required for HAT activity, thus affecting post-translational modification of $\mathrm{K} 27$ on $\mathrm{H} 3$ variants [8]. Interestingly, EP300 D1399Y mutations alter its interaction with transcription factor AP-2alpha indirectly leading to the transactivation of Myc [16]. Moreover, the tumor harboring the EP300 mutation was located in the thalamus which is a neuroanatomical structure in the brain midline where the majority of HGGs harbor H3K27M mutations. This novel epigenetic mutation may reproduce some of the effects of K27M in a wildtype H3K27 tumor. In our study, the tumor with the EP300 D1399N mutation had increased Myc expression (data not shown), suggesting that this particular EP300 mutation may also play a role in Mycrelated oncogenesis similar to $\mathrm{K} 27 \mathrm{M}$ mutagenesis. Although interesting, these findings need further testing and functional validation in relevant disease models. The two HGGs from patients with germline NF1 did not show a high mutational burden at diagnosis or at recurrence, and no clear associated driver mutation. Interestingly, a tendency towards increased copy number alteration was observed in both pairs at recurrence. These findings also need further validation on a larger sample set.

Somatic mutations in RTKs are common in adult GBM $[5,6]$ and are generally found at low frequencies in pHGGs [41]. Similar to our previous report [41], the H3/IDH1 wildtype group in this study seemed enriched 
with RTK mutations $(5 / 7,71 \%)$. One striking finding in this molecular group was the discovery of EGFR missense mutations in the primary occurrence of HGG10 (T790M and E709A), which were lost in the recurrence. A shared EGFR R222C missense mutation was present in both the primary and recurrent tumors, indicating that alteration of the RTK pathway is nonetheless conserved in the recurrent tumor. The EGFR T790M mutation has been implicated in acquired resistance to most EGFR tyrosine kinase inhibitors [21, 27]. This may, in part, explain tumor progression in this patient despite treatment with lapatinib (Novartis, East Hanover, NJ), and highlights the importance of identifying resistancepromoting mutations in the clinical setting. We also identified three tumors with targetable RTK lesions (PDGFRA, ERBB2, ERBB 4) that were exclusive to the recurrent tumor (HGG5, HG8, HGG11), indicating that genomic data from tumor tissue at recurrence may provide better guidance for therapeutic choices. Conversely, one case harbored a low level subclonal PIK3CA mutation that was discovered by a clinical genomics panel in the primary tumor, but was not identified by WES in different primary tumor blocks from the same case, nor in multiple samplings of the recurrent tumor. Excluding the subclonal nature of this mutation, and confirming its maintenance at recurrence are important therapeutic considerations before embarking on targeted treatment, especially with single agents such as rapamycin used in this patient.

\section{Conclusions}

In conclusion, this study further highlights the molecular distinction between pediatric and adult HGGs, especially in therapy-induced tumor evolution. We show that genes with driver mutations (H3, TP53, PPMID, ZMYND11, $E P 300)$ as well as some targetable mutations (e.g. $I D H 1$, $B R A F \mathrm{~V} 600 \mathrm{E})$ are conserved. Importantly, we demonstrate that some actionable mutations are unstable (PI3K, $E G F R)$, indicating that re-biopsy is warranted in order to optimize personalized therapy. The presence of subclonal targetable alterations concurrently with driver mutations supports the use of combination therapy approaches to address disease biology and evolution with the aim of improving patient outcomes.

\section{Additional files}

Additional file 1: Table S1. Tissue location and type, tumor content, WES coverage and digital droplet PCR results in 16 pairs of pHGG analyzed in this study. (XLSX $13 \mathrm{~kb}$ )

Additional file 2: Table S2. Somatic and putative somatic mutation information for 16 pairs of pHGGs analyzed in this study. (XLSX $301 \mathrm{~kb}$ )
Additional file 3: Figure S1. IGV views a subclonal low frequency PIK3CA mutation in HGG3 from a clinical sequencing panel, WES, and targeted sequencing. (PDF $2380 \mathrm{~kb}$ )

Additional file 4: Table S3. Number of Single Nucleotide Variants (SNVs) and regions of Allelic Imbalance (Al) present in tumors as shared, primary only, or recurrence only in the $\mathrm{PHGG}$ tumor pairs analyzed in this study. (XLSX 13 kb)

Additional file 5: Figure S2. Percentages of SNVs and regions of Allelic Imbalance as shared, primary only and recurrence only. (PDF 908 kb)

Additional file 6: Figure S3. Immunohistochemical staining for the MMR panel (MLH1, MSH2, MSH6 and PMS2) in the HGG11 primary tumor. (PDF $23521 \mathrm{~kb}$ )

Additional file 7: Figure S4. Genome-wide view of copy number variations in HGG9 primary and recurrence tumors calculated from Whole Exome Sequencing data. (PDF $2757 \mathrm{~kb}$ )

Additional file 8: Table S4. Chromosomal location of Al segments in 15 pairs of pHGG analyzed in this study. (XLSX $61 \mathrm{~kb}$ )

Additional file 9: Table S5. Copy number variation (CNV) segments in primary and recurrence tumors from 8 of 16 pairs of pHGG with matched normal tissue available. (XLSX $71 \mathrm{~kb})$

\section{Abbreviations}

IDH1: isocitrate dehydrogenase 1; TP53: tumor protein 53; ACVR1: activin a receptor, type I; ZMYND11: zinc finger MYND domain-containing protein 11; EP300: histone acetyltransferase p300; BRAF: b-raf proto-oncogene;

NF-1: neurofibromatosis 1; ATRX: alpha-thalassemia/mental retardation syndrome, Nondeletion type, $x$-linked; EGFR: epidermal growth factor receptor; ERBB2: Erb-B2 receptor tyrosine kinase 2; PDGFRA: platelet derived growth factor receptor alpha; PI3K: phosphoinositide 3-kinase

\section{Acknowledgments}

We would like to thank all the patients and families who have supported this project. We would also like to thank Dr. Blake Gilks for his expert consultation of the immunohistochemical interpretation. This research was supported by: Cancer Free KIDS (RS), US National Institutes of Health (NIH) grant P01CA196539 (NJ, JM), the Canadian Institutes for Health Research (CIHR) grant MOP 286756 to NJ; the Fonds de recherche du Québec Santé (FRQS) to CK. JM holds a Canada Research Chair (tier 2). Computational infrastructure was provided by Compute Canada. This work was performed within the context of the I-CHANGE consortium and supported by funding from Genome Canada, Genome Quebec, the Institute for Cancer Research of the CIHR, McGill University and the Montreal Children's Hospital Foundation. $\mathrm{NJ}$ is a member of the Penny Cole Laboratory and the recipient of a Chercheur Boursier, Chaire de Recherche Award from the FRQS. MKM is funded by a CIHR Banting postdoctoral fellowship, NDJ is a recipient of an FRQS studentship.

\section{Ethics approval and consent to participate}

All procedures performed in studies involving human participants were in accordance with the ethical standards of the institutional and/or national research committee and with the 1964 Helsinki declaration and its later amendments or comparable ethical standards.

\section{Consent for publication}

Informed consent was obtained from all individual participants included in this study.

\section{Competing interests}

The authors declare that they have no competing interests.

\section{Publisher's Note}

Springer Nature remains neutral with regard to jurisdictional claims in published maps and institutional affiliations.

\section{Author details}

'Brain Tumor Center, Cincinnati Children's Hospital Medical Center, 3333 Burnet Avenue, Cincinnati, OH 45229, USA. ²Department of Human Genetics, McGill University, Montreal, QC H3A 1B1, Canada. ${ }^{3}$ Department of Pediatrics, McGill University and McGill University Heath Centre Research Institute, 
Montreal, QC H4A 3J1, Canada. ${ }^{4}$ Department of Laboratory Medicine and Pathology, Nationwide Children's Hospital, and the Ohio State University, Columbus, OH 43205, USA. ${ }^{5}$ Division of Hematology/Oncology and Bone Marrow Transplantation, Nationwide Children's Hospital, Columbus, $\mathrm{OH}$ 43205, USA. ${ }^{6}$ Department of Pathology, Montreal Neurological Hospital, McGill University, Montreal, QC H3A 2B4, Canada. 'The Lady Davis Institute, Jewish General Hospital, Montreal, QC H3T 1E2, Canada.

\section{Received: 4 October 2017 Accepted: 4 October 2017}

\section{Published online: 30 October 2017}

\section{References}

1. Bai H, Harmanci AS, Erson-Omay EZ, Li J, Coskun S, Simon M et al (2016) Integrated genomic characterization of IDH1-mutant glioma malignant progression. Nat Genet 48:59-66. doi: 10.1038/ng.3457

2. Balss J, Meyer J, Mueller W, Korshunov A, Hartmann C, von Deimling A (2008) Analysis of the IDH1 codon 132 mutation in brain tumors. Acta Neuropathol 116:597-602. doi: 10.1007/s00401-008-0455-2

3. Beltran H, Prandi D, Mosquera JM, Benelli M, Puca L, Cyrta J et al (2016) Divergent clonal evolution of castration-resistant neuroendocrine prostate cancer. Nat Med 22:298-305. doi: 10.1038/nm.4045

4. Bouffet $\mathrm{E}$, Larouche V, Campbell BB, Merico D, de Borja R, Aronson M et al (2016) Immune checkpoint inhibition for Hypermutant Glioblastoma Multiforme resulting from Germline Biallelic mismatch repair deficiency. J Clin Oncol 34:2206-2211. doi: 10.1200/jco.2016.66.6552

5. Brennan CW, Verhaak RG, McKenna A, Campos B, Noushmehr H, Salama SR et al (2013) The somatic genomic landscape of glioblastoma. Cell 155:462-477. doi: 10.1016/j.cell.2013.09.034

6. Cancer Genome Atlas Research N (2008) Comprehensive genomic characterization defines human glioblastoma genes and core pathways. Nature 455:1061-1068. doi: 10.1038/nature07385

7. Cerami E, Gao J, Dogrusoz U, Gross BE, Sumer SO, Aksoy BA et al (2012) The cBio cancer genomics portal: an open platform for exploring multidimensional cancer genomics data. Cancer Discov 2:401-404 doi: 10.1158/2159-8290.CD-12-0095

8. Delvecchio M, Gaucher J, Aguilar-Gurrieri C, Ortega E, Panne D (2013) Structure of the p300 catalytic core and implications for chromatin targeting and HAT regulation. Nat Struct Mol Biol 20:1040-1046. doi: 10.1038/nsmb.2642

9. Eckel-Passow JE, Lachance DH, Molinaro AM, Walsh KM, Decker PA, Sicotte $\mathrm{H}$ et al (2015) Glioma groups based on 1p/19q, IDH, and TERT promoter mutations in tumors. N Engl J Med 372:2499-2508. doi: 10.1056/ NEJMoa1407279

10. Eckert A, Kloor M, Giersch A, Ahmadi R, Herold-Mende C, Hampl JA et al (2007) Microsatellite instability in pediatric and adult high-grade gliomas. Brain Pathol 17:146-150. doi: 10.1111/j.1750-3639.2007.00049.x

11. Eirew P, Steif A, Khattra J, Ha G, Yap D, Farahani H et al (2015) Dynamics of genomic clones in breast cancer patient xenografts at single-cell resolution. Nature 518:422-426. doi: 10.1038/nature13952

12. Fontebasso A, Schwartzentruber J, Khuong-Quang D-A, Liu X-Y, Sturm D, Korshunov A et al (2013) Mutations in SETD2 and genes affecting histone H3K36 methylation target hemispheric high-grade gliomas. Acta Neuropathol 125:659-669. doi: 10.1007/s00401-013-1095-8

13. Fontebasso AM, Gayden T, Nikbakht H, Neirinck M, Papillon-Cavanagh S, Majewski J et al (2014) Epigenetic dysregulation: a novel pathway of oncogenesis in pediatric brain tumors. Acta Neuropathol 128:615-627. doi: 10.1007/s00401-014-1325-8

14. Fontebasso AM, Papillon-Cavanagh S, Schwartzentruber J, Nikbakht $\mathrm{H}_{\text {, }}$ Gerges N, Fiset PO et al (2014) Recurrent somatic mutations in ACVR1 in pediatric midline high-grade astrocytoma. Nat Genet 46:462-466. doi: 10.1038/ng.2950

15. Gao J, Aksoy BA, Dogrusoz U, Dresdner G, Gross B, Sumer SO et al (2013) Integrative analysis of complex cancer genomics and clinical profiles using the cBioPortal. Sci Signal 6:pl1. doi: 10.1126/scisignal.2004088

16. Gaubatz S, Imhof A, Dosch R, Werner O, Mitchell P, Buettner R et al (1995) Transcriptional activation by Myc is under negative control by the transcription factor AP-2. EMBO J 14:1508-1519

17. Guo R, Zheng L, Park JW, Lv R, Chen H, Jiao F et al (2014) BS69/ZMYND11 reads and connects histone $\mathrm{H} 3.3$ lysine 36 trimethylation-decorated chromatin to regulated pre-mRNA processing. Mol Cell 56:298-310. doi: 10.1016/j.molcel.2014.08.022
18. Hoffman LM, DeWire M, Ryall S, Buczkowicz P, Leach J, Miles L et al (2016) Spatial genomic heterogeneity in diffuse intrinsic pontine and midline highgrade glioma: implications for diagnostic biopsy and targeted therapeutics. Acta Neuropathol Commun 4:1. doi: 10.1186/s40478-015-0269-0

19. Johnson BE, Mazor T, Hong C, Barnes M, Aihara K, McLean CY et al (2014) Mutational analysis reveals the origin and therapy-driven evolution of recurrent glioma. Science 343:189-193. doi: 10.1126/science.1239947

20. Jones C, Karajannis MA, Jones DTW, Kieran MW, Monje M, Baker SJ et al (2017) Pediatric high-grade glioma: biologically and clinically in need of new thinking. Neuro-Oncology 19:153-161. doi: 10.1093/neuonc/now101

21. Kobayashi S, Boggon TJ, Dayaram T, Janne PA, Kocher O, Meyerson M et al (2005) EGFR mutation and resistance of non-small-cell lung cancer to gefitinib. N Engl J Med 352:786-792. doi: 10.1056/NEJMoa044238

22. Korbel JO, Campbell PJ (2013) Criteria for inference of chromothripsis in cancer genomes. Cell 152:1226-1236. doi: 10.1016/j.cell.2013.02.023

23. Le DT, Uram JN, Wang H, Bartlett BR, Kemberling H, Eyring AD et al (2015) PD-1 blockade in tumors with mismatch-repair deficiency. N Engl J Med 372:2509-2520. doi: 10.1056/NEJMoa1500596

24. Lewis PW, Muller MM, Koletsky MS, Cordero F, Lin S, Banaszynski LA et al (2013) Inhibition of PRC2 activity by a gain-of-function $\mathrm{H} 3$ mutation found in pediatric glioblastoma. Science 340:857-861. doi: 10.1126/science.1232245

25. Liu X, Wang L, Zhao K, Thompson PR, Hwang Y, Marmorstein R et al (2008) The structural basis of protein acetylation by the p300/CBP transcriptional coactivator. Nature 451:846-850. doi: 10.1038/nature06546

26. Liu XY, Gerges N, Korshunov A, Sabha N, Khuong-Quang DA, Fontebasso AM et al (2012) Frequent ATRX mutations and loss of expression in adult diffuse astrocytic tumors carrying IDH1/IDH2 and TP53 mutations. Acta Neuropathol 124:615-625. doi: 10.1007/s00401-012-1031-3

27. Lynch TJ, Bell DW, Sordella R, Gurubhagavatula S, Okimoto RA, Brannigan BW et al (2004) Activating mutations in the epidermal growth factor receptor underlying responsiveness of non-small-cell lung cancer to gefitinib. N Engl J Med 350:2129-2139. doi: 10.1056/NEJMoa040938

28. Morrissy AS, Garzia L, Shih DJ, Zuyderduyn S, Huang X, Skowron P et al (2016) Divergent clonal selection dominates medulloblastoma at recurrence. Nature 529:351-357. doi: 10.1038/nature16478

29. Nadaf J, Majewski J, Fahiminiya S (2015) ExomeAl: detection of recurrent allelic imbalance in tumors using whole-exome sequencing data. Bioinformatics 31:429-431. doi: 10.1093/bioinformatics/btu665

30. Nikbakht H, Panditharatna E, Mikael LG, Li R, Gayden T, Osmond M et al (2016) Spatial and temporal homogeneity of driver mutations in diffuse intrinsic pontine glioma. Nat Commun 7:11185. doi: 10.1038/ncomms11185

31. Northcott PA, Buchhalter I, Morrissy AS, Hovestadt V, Weischenfeldt J, Ehrenberger T et al (2017) The whole-genome landscape of medulloblastoma subtypes. Nature 547:311-317. doi: 10.1038/nature22973

32. Papillon-Cavanagh S, Lu C, Gayden T, Mikael LG, Bechet D, Karamboulas C et al (2017) Impaired H3K36 methylation defines a subset of head and neck squamous cell carcinomas. Nat Genet 49:180-185. doi: 10.1038/ng.3757

33. Pasini D, Malatesta M, Jung HR, Walfridsson J, Willer A, Olsson L et al (2010) Characterization of an antagonistic switch between histone $\mathrm{H3}$ lysine 27 methylation and acetylation in the transcriptional regulation of Polycomb group target genes. Nucleic Acids Res 38:4958-4969. doi: 10.1093/nar/gkq244

34. Pasqualucci L, Dominguez-Sola D, Chiarenza A, Fabbri G, Grunn A, Trifonov $\checkmark$ et al (2011) Inactivating mutations of acetyltransferase genes in B-cell lymphoma. Nature 471:189-195. doi: 10.1038/nature09730

35. Paugh BS, Qu C, Jones C, Liu Z, Adamowicz-Brice M, Zhang J et al (2010) Integrated molecular genetic profiling of pediatric high-grade gliomas reveals key differences with the adult disease. J Clin Oncol 28:3061-3068. doi: 10.1200/JCO.2009.26.7252

36. Ramaswamy V, Remke M, Bouffet E, Faria CC, Perreault S, Cho YJ et al (2013) Recurrence patterns across medulloblastoma subgroups: an integrated clinical and molecular analysis. Lancet Oncol 14:1200-1207. doi: 10.1016/ S1470-2045(13)70449-2

37. Schwartzentruber J, Korshunov A, Liu X-Y, Jones DTW, Pfaff E, Jacob K et a (2012) Driver mutations in histone H3.3 and chromatin remodelling genes in paediatric glioblastoma. Nature 482:226-231. doi: 10.1038/nature10833

38. Shi Y, Majewski J (2013) FishingCNV: a graphical software package for detecting rare copy number variations in exome-sequencing data. Bioinformatics 29:1461-1462. doi: 10.1093/bioinformatics/btt151

39. Siravegna G, Mussolin B, Buscarino M, Corti G, Cassingena A, Crisafulli G et a (2015) Clonal evolution and resistance to EGFR blockade in the blood of colorectal cancer patients. Nat Med 21:795-801. doi: 10.1038/nm.3870 
40. Staedtke V, Bai RY, Laterra J (2016) Investigational new drugs for brain cancer. Expert Opin Investig Drugs 25:937-956. doi: 10.1080/13543784.2016.1182497

41. Sturm D, Witt H, Hovestadt V, Khuong-Quang D-A, Jones David TW, Konermann C et al (2012) Hotspot mutations in H3F3A and IDH1 define distinct epigenetic and biological subgroups of Glioblastoma. Cancer Cell 22:425-437. doi: 10.1016/..ccr.2012.08.024

42. Taylor KR, Mackay A, Truffaux N, Butterfield YS, Morozova O, Philippe C et al (2014) Recurrent activating ACVR1 mutations in diffuse intrinsic pontine glioma. Nat Genet 46:457-461. doi: 10.1038/ng.2925

43. Van der Auwera GA, Carneiro MO, Hartl C, Poplin R, Del Angel G, LevyMoonshine A et al (2013) From FastQ data to high confidence variant calls: the genome analysis toolkit best practices pipeline. Curr Protoc Bioinformatics 43:11.10.1-11.1033. doi: 10.1002/0471250953.bi1110s43

44. Viana-Pereira M, Lee A, Popov S, Bax DA, Al-Sarraj S, Bridges LR et al (2011) Microsatellite instability in pediatric high grade glioma is associated with genomic profile and differential target gene inactivation. PLoS One 6: e20588. doi: 10.1371/journal.pone.0020588

45. Wang J, Cazzato E, Ladewig E, Frattini V, Rosenbloom DI, Zairis S et al (2016) Clonal evolution of glioblastoma under therapy. Nat Genet 48:768-776. doi: 10.1038/ng.3590

46. Wang K, Li M, Hakonarson H (2010) ANNOVAR: functional annotation of genetic variants from high-throughput sequencing data. Nucleic Acids Res 38:e164. doi: 10.1093/nar/gkq603

47. Wang X, Dubuc AM, Ramaswamy V, Mack S, Gendoo DM, Remke M et a (2015) Medulloblastoma subgroups remain stable across primary and metastatic compartments. Acta Neuropathol 129:449-457. doi: 10.1007/ s00401-015-1389-0

48. Wang Y, Waters J, Leung ML, Unruh A, Roh W, Shi X et al (2014) Clonal evolution in breast cancer revealed by single nucleus genome sequencing. Nature 512:155-160. doi: 10.1038/nature13600

49. Wen H, Li Y, Xi Y, Jiang S, Stratton S, Peng D et al (2014) ZMYND11 links histone H3.3K36me3 to transcription elongation and tumour suppression. Nature 508:263-268. doi: 10.1038/nature13045

50. Wu G, Broniscer A, McEachron TA, Lu C, Paugh BS, Becksfort J et al (2012) Somatic histone $\mathrm{H} 3$ alterations in pediatric diffuse intrinsic pontine gliomas and non-brainstem glioblastomas. Nat Genet 44:251-253. doi: 10.1038/ng.1102

51. Wu G, Diaz AK, Paugh BS, Rankin SL, Ju B, Li Y et al (2014) The genomic landscape of diffuse intrinsic pontine glioma and pediatric non-brainstem high-grade glioma. Nat Genet 46:444-450. doi: 10.1038/ng.2938

52. Yan H, Parsons DW, Jin G, McLendon R, Rasheed BA, Yuan W et al (2009) IDH1 and IDH2 mutations in gliomas. N Engl J Med 360:765-773. doi: 10. 1056/NEJMoa0808710

53. Yuan $L$, Chi Y, Chen W, Chen X, Wei P, Sheng W et al (2015) Immunohistochemistry and microsatellite instability analysis in molecular subtyping of colorectal carcinoma based on mismatch repair competency. Int J Clin Exp Med 8:20988-21000

54. Zhang CZ, Leibowitz ML, Pellman D (2013) Chromothripsis and beyond: rapid genome evolution from complex chromosomal rearrangements. Genes Dev 27:2513-2530. doi: 10.1101/gad.229559.113

55. Zhang L, Chen LH, Wan H, Yang R, Wang Z, Feng J et al (2014) Exome sequencing identifies somatic gain-of-function PPM1D mutations in brainstem gliomas. Nat Genet 46:726-730. doi: 10.1038/ng.2995

\section{Submit your next manuscript to BioMed Central and we will help you at every step:}

- We accept pre-submission inquiries

- Our selector tool helps you to find the most relevant journal

- We provide round the clock customer support

- Convenient online submission

- Thorough peer review

- Inclusion in PubMed and all major indexing services

- Maximum visibility for your research

Submit your manuscript at www.biomedcentral.com/submit

) Biomed Central 